\title{
$\beta$-catenin is regulated by USP9x and mediates resistance to TRAIL-induced apoptosis in breast cancer
}

\author{
WEN OUYANG ${ }^{1 *}$, SHIMIN ZHANG ${ }^{1 *}$, BO YANG $^{1}$, CHUNXU YANG $^{1}$, \\ JUNHONG ZHANG ${ }^{1}$, FUXIANG ZHOU ${ }^{2}$ and CONGHUA XIE ${ }^{1,2}$ \\ ${ }^{1}$ Department of Radiation and Medical Oncology and ${ }^{2}$ Hubei Key Laboratory of Tumor Biological Behaviors, \\ Zhongnan Hospital, Wuhan University, Wuhan, Hubei 430071, P.R. China
}

Received July 22, 2015; Accepted September 4, 2015

DOI: $10.3892 /$ or.2015.4463

\begin{abstract}
To investigate the regulatory mechanisms of decoy receptor expression in TRAIL-resistant breast cancer MCF-7 cells, cytotoxicity and apoptosis assays were applied to examine sensitivity to TRAIL in breast cancer cells. Immunofluorescence and immunoprecipitation were used to detect the co-localization and interaction of USP9x and $\beta$-catenin. Luciferase assay was used to examine activity of the DcR1/DcR2/OPG reporter. Overexpression/silencing of $\beta$-catenin was performed to confirm $\beta$-catenin mediated transcription of the decoy receptors. Additionally, silencing of USP9x was performed to prove that USP9X stabilizes $\beta$-catenin and mediates TRAIL-resistance. It was found that USP9 $x$ interacted with $\beta$-catenin and inhibited the degradation of $\beta$-catenin through the deubiquitination of $\beta$-catenin. Luciferase reporter assays showed induction of DcR1/DcR2/OPG reporter activity observed upon co-transfection of $\beta$-catenin and Tcf- 4 . The overexpression/silencing of $\beta$-catenin further confirmed the role of $\beta$-catenin in the regulation of transcription of the decoy receptors. Silencing of USP9x directly evidenced that USP9x affected the protein expression level of $\beta$-catenin, the transcription level of the decoy receptors, and reversed TRAIL-resistance of MCF-7 cells. In conclusion, USP9x interacted with and stabilized $\beta$-catenin through deubiquitination to mediate transcription of the decoy receptors in breast cancer cells. Our results offer new insights into the mechanisms of resistance to TRAIL, and USP9 $x$ could potentially be a therapeutic target for TRAILresistant breast cancers.
\end{abstract}

Correspondence to: Dr Conghua Xie, Department of Radiation and Medical Oncology, Zhongnan Hospital, Wuhan University, 169 Donghu Road, Wuchang, Wuhan, Hubei 430071, P.R. China

E-mail: chxie_65@hotmail.com

*Contributed equally

Key words: USP9x, $\beta$-catenin, deubiquitination, TRAIL, resistance

\section{Introduction}

Tumor necrosis factor-related apoptosis ligand (TRAIL) is a member of the tumor necrosis factor (TNF) superfamily that includes cytokines such as TNF and FasL. Comparing with TNF and FasL, TRAIL is capable of selectively inducing apoptosis of transformed or tumor cells, but not normal cells (1). This feature has greatly promoted the development of TRAIL-based cancer targeted therapy. Accumulating preclinical studies demonstrate that TRAIL can effectively induce apoptosis of a variety of cancer cell lines, and inhibit the growth of xenografted tumors (2). Currently, completed and ongoing phase I/II clinical trials using recombinant TRAIL proteins or agonistic antibodies of TRAIL receptor show encouraging outcomes without significant toxicity $(3,4)$. Consequently, TRAIL is a promising target agent for the treatment of human cancer.

There are five TRAIL receptors, including death receptor DR4 and DR5, decoy receptor DcR1, DcR2 and osteoprotegerin (OPG) (5). TRAIL induces apoptosis through interacting with its death receptors. Both DR4 and DR5 contain a conserved death domain (DD) motif for initiating apoptosis (5). Unlike DR4 and DR5, DcR1 lacks an intracellular domain, while DcR2 contains a truncated and non-functional DD, and OPG is a soluble receptor at low affinity to TRAIL. The apoptotic pathway cannot be engaged after TRAIL binds to the decoy receptors (5). Thus, these decoy receptors act to antagonize TRAIL-induced apoptosis by competing for ligand binding. This competition for binding to TRAIL is thought to be the mechanism that TRAIL selectively kills tumor or transformed cells but not most normal cells. As a result, the proportion of death receptors and decoy receptors on cellular membrane is involved in sensitivity to TRAIL. For example, certain cancer cells with high expression levels of DcRl and DcR2 show resistance to TRAIL-induced apoptosis (6). Therefore, it is reasonable for us to believe that alteration of TRAIL receptors via genetic and epigenetic changes can affect sensitivity to TRAIL in cells. Our previous results also confirmed that radiation was able to improve TRAIL-induced apoptosis in laryngeal cancer cells by upregulating the expression of DR5 (7). In addition, it was reported that some chemotherapy agents could improve the cancer cell TRAIL-sensitivity through increasing the expression level of death receptors (8). 
However, the molecular alterations of sensitization to TRAIL in cancer cells have been mostly studied in the context of elevated death receptors' levels, little on decoy receptors. Whereas, decoy receptors are widely expressed in cancer and normal cells. Therefore, reversing TRAIL-resistance in cancer cells has become a new research direction. Thus, understanding the regulatory mechanisms of the decoy receptor expression can help us to develop TRAIL-based agents for the treatment of human cancer.

It is known that $\mathrm{Wnt} / \beta$-catenin signaling pathway plays a key role in development, tissue homeostasis and cancer (9). $\beta$-catenin is a central mediator of $\mathrm{Wnt} / \beta$-catenin signaling pathway (10). $\beta$-catenin controls the transcription of Wnt target genes during both normal and malignant development (9). In the presence of $\mathrm{Wnt} / \beta$-catenin signaling, cytoplasmic $\beta$-catenin is stabilized and accumulated in a hypo-phosphorylated form, then translocates into the nucleus where it interacts with T-cell factor/lymphoid enhancing factor (TCF/LEF) to form a transcriptional complex that induces the expression of target genes, such as c-myc, Jun, Anxa-2, and CD44 $(9,11)$. Analyzing the gene sequence of decoy receptors DcR1, DcR2, and OPG, we found that their gene promoter upstream regions are enriched in binding sites for TCF/LEF transcription factor. It is reasonable for us to assume that the interaction of $\beta$-catenin and TCF/LEF could activate the transcription of DcR1, DcR2 and OPG, so the expression and distribution of $\beta$-catenin was correlated with sensitivity to TRAIL in cancer cells. There are two studies partly confirming this, Glass et al found that $\beta$-catenin together with TCF proteins could regulate expression of OPG in osteoblast (12); De Toni et al showed that production of OPG was regulated by $\beta$-catenin/Tcf- 4 in colorectal cancer cells, and accumulation of OPG in medium of cultivated cells caused resistance to TRAIL (13).

However, the mechanisms of $\beta$-catenin overexpression in breast cancer cells remained elusive. Wnt/ $\beta$-catenin signaling pathway is absent in mature normal cells most of the time, so cytoplasmic $\beta$-catenin is maintained at low levels through degradation by the ubiquitin-proteasome pathway (14). However, the ubiquitination of substrates is a reversible reaction, and the ubiquitinated substrates can be deubiquitinated by so-called deubiquitinating enzymes. So deubiquitination plays a negative regulatory role in the process of ubiquitin-mediated protein degradation, and the increased deubiquitinating enzymes may improve the stability of some growth factors and signal transduction molecules which are degraded by ubiquitin-proteasome pathway (15). USP9 $x$ is a member of the USP (ubiquitin specific peptidase) family, a human deubiquitinating enzyme. Fam is the homologous protein of USP9x in mouse. Taya et al found that overexpression of Fam in mouse L cells resulted in an elevation of the level of $\beta$-catenin and an elongation of the half-life of $\beta$-catenin, and Fam was colocalized with $\beta$-catenin in the cytoplasm in these L cells (16). Here, we hypothesized that USP9x interacted with and stabilized $\beta$-catenin through deubiquitination, resulting in overexpression of decoy receptors and TRAIL-resistance in breast cancer cells.

\section{Materials and methods}

Cell culture. Human breast cancer cell lines including MCF-7 and MDA-MB-231, and HEK293T were obtained directly from the Type Culture Collection of the Chinese Academy of Sciences (Shanghai, China), where cell lines have been tested and authenticated, and all cell lines were passaged in our laboratory for less than 6 months after resuscitation. The breast cancer cell lines MCF-7 and MDA-MB-231 were cultured in RPMI-1640 medium, while HEK293T cells were cultured in Dulbecco's modified Eagle's medium (DMEM) (both from Hyclone, USA) at $37^{\circ} \mathrm{C}$ in a humidified atmosphere of $5 \%$ $\mathrm{CO}_{2}$. The media were supplemented with $10 \%$ heat-inactivated fetal bovine serum and $1 \%$ penicillin/streptomycin, and were changed every 3-5 days. Cells in log-phase growth were harvested by trypsinization for use in various assays.

Antibodies and reagents. Recombinant soluble TRAIL (rhTRAIL), designed for clinical use, was obtained from Shanghai TRAIL Bio-Technical Co., Ltd. Monoclonal antibodies to decoy receptors DcR1, DcR2, and OPG, and $\beta$-catenin, USP9x were purchased from Abcam (Cambridge, MA, USA). Monoclonal antibody to GAPDH was from Santa Cruz Biotechnology Inc. Fluorescein (FITC)-conjugated goat anti-rabbit IgG and rhodamine (TRITC)-conjugated goat anti-mouse IgG were from Beijing Biosynthesis Biotechnology Co., Ltd. Horseradish peroxidase (HRP)-conjugated goat anti-mouse $\mathrm{IgG}$ and goat anti-rabbit $\mathrm{IgG}$ were from Southern Biotech. Protease inhibitor mixture, Triton X-100, Tween-20 and other chemicals of analytic grade were purchased from Sigma-Aldrich.

Plasmids, siRNA and transfection. For plasmid construction, $\beta$-catenin cDNA was amplified from the total mRNA of MCF-7 cells by RT-PCR and was subcloned into pCMV-C-Flag vector (Clontech Laboratories, Mountain View, CA, USA), yielding a construct for expression of a fusion protein with a Flag tag at the $\mathrm{C}$-terminus of $\beta$-catenin. The construct was verified by automated DNA sequencing.

For small interfering RNA (siRNA) oligonucleotides, USP9x, $\beta$-catenin and negative control FAM siRNA oligonucleotides were obtained from Shanghai GenePharma Co., Ltd. (Shanghai, China). The negative control FAM siRNA with green fluorescence was used as a control.

For transfection, cells were seeded into 6-well plates (Corning Inc., Corning, NY, USA) without antibiotics. After $24 \mathrm{~h}$, transfections were carried out using Lipofectamine 2000 reagent (Invitrogen, Carlsbad, CA, USA) according to the manufacturer's instructions. The efficiency of transfection was evaluated by fluorescence intensity using inverted fluorescence microscopy (Nikon, Japan).

Cytotoxicity assays. Cytotoxicity assays and cell survival assays were determined by the Cell Counting Kit-8 (CCK-8) assay. Briefly, cells were plated at 2,000/well in 96-well plates (Corning Inc.). The next day, cells (confluence 80-90\%) were treated with indicated concentrations of rhTRAIL and incubated for $4 \mathrm{~h}$. Depending on the needs of the experiment, cells were transfected or not with USP $9 x / \beta$-catenin overexpression plasmid/siRNA before adding rhTRAIL. At the end of the assay, the culture medium was removed, then a mixture of fresh medium (without phenol red) containing CCK-8 (Sigma-Aldrich) was added and incubated for $1 \mathrm{~h}$. The absorbance was determined using a microplate reader at $450 \mathrm{~nm}$ 
(Tecan, Port Melbourne, VIC, Australia). Cell viability was defined as the relative absorbance of treated versus untreated cells. All assays were performed in five replicates and repeated at least three times.

Apoptosis assays. Cells were grown on 6-well plates to $70-80 \%$ confluence and treated with transfection and/or indicated concentrations of rhTRAIL incubation. First, cells were pretreated with the caspase inhibitor ZVAD-fmk for $2 \mathrm{~h}$ to block endogenous TRAIL-induced apoptosis, then at selected time-points, cells were collected and incubated with $5 \mu \mathrm{l}$ Annexin V-FITC (BestBio, Shanghai, China) for $15 \mathrm{~min}$ and $10 \mu \mathrm{l}$ propidium iodide (BestBio Shanghai, China) for $5 \mathrm{~min}$ in the dark at $4^{\circ} \mathrm{C}$. The apoptotic profile was obtained by flow cytometry immediately afterwards (Beckman Coulter, Miami, FL, USA).

Immunofluorescence. Cells were cultured on glass chamber slides to $30-50 \%$ confluence. First, cells were fixed with $4 \%$ PBS-buffered paraformaldehyde for $20 \mathrm{~min}$, washed with PBS three times, and blocked with $1 \%$ goat serum for $1 \mathrm{~h}$. Afterwards, cells were counterstained with anti- $\beta$-catenin rabbit monoclonal antibody and anti-USP9x mouse monoclonal antibody at $4^{\circ} \mathrm{C}$ overnight, then counterstained with FITC-conjugated goat anti-rabbit antibody and TRITC-conjugated goat anti-mouse antibody at room temperature in the dark for $1 \mathrm{~h}$. Then the cell nucleus was stained with $1 \mu \mathrm{g} / \mathrm{ml}$ DAPI at room temperature in the dark for $15 \mathrm{~min}$. Immunofluorescence was analyzed using a confocal laser-scanning microscope (Leica, Wetzlar, Germany).

Immunoprecipitation. $\beta$-catenin was overexpressed in HEK293T cell lines. Cells were harvested and cell lysates were cleared by centrifugation at $12,000 \mathrm{x}$ g for $30 \mathrm{~min}$, then $2 \mathrm{mg}$ primary antibody (anti-USP9X antibody or anti- $\beta$-catenin antibody) and $20 \mu \mathrm{l}$ of resuspended volume of Protein $\mathrm{A} / \mathrm{G}$ PLUS-Agarose were added and incubated at $4{ }^{\circ} \mathrm{C}$ on a rocker platform overnight. Then immunoprecipitates were collected by centrifugation at $2,500 \mathrm{rpm}$ for $5 \mathrm{~min}$ at $4^{\circ} \mathrm{C}$ and washed 4 times with $1 \mathrm{ml}$ RIPA buffer, each time repeating centrifugation step as above. After the final wash, the supernatant was discarded and the pellet was resuspend in $40 \mu \mathrm{l}$ of $1 \mathrm{X}$ electrophoresis sample buffer for immunoblotting.

Luciferase assay. For reporter gene assays in HEK293T cells, cells were transfected with $200 \mathrm{ng}$ of a luciferase reporter gene construct for DcR1/DcR2/OPG, and an expression construct of Tcf4 and $\beta$-catenin, and $200 \mathrm{ng}$ of constitutively active $\beta$-galactosidase reporter plasmid pCH110 (Amersham Pharmacia Biosciences). Transfections were done as recommended by the manufacturer, and luciferase activity was measured $48 \mathrm{~h}$ later (Promega, Madison, WI, USA) according to the manufacturer's instructions using a luminescence reader (Tecan). To normalize luciferase activity, $\beta$-galactosidase activity was determined using standard methods.

Reverse transcription-PCR. RNA was isolated using the TRIzol reagent (Invitrogen). RT-PCR was carried out using the high-fidelity RT-PCR kit (Invitrogen) as per the manufacturer's instructions. Primer pairs used for detection of DcR 1
A

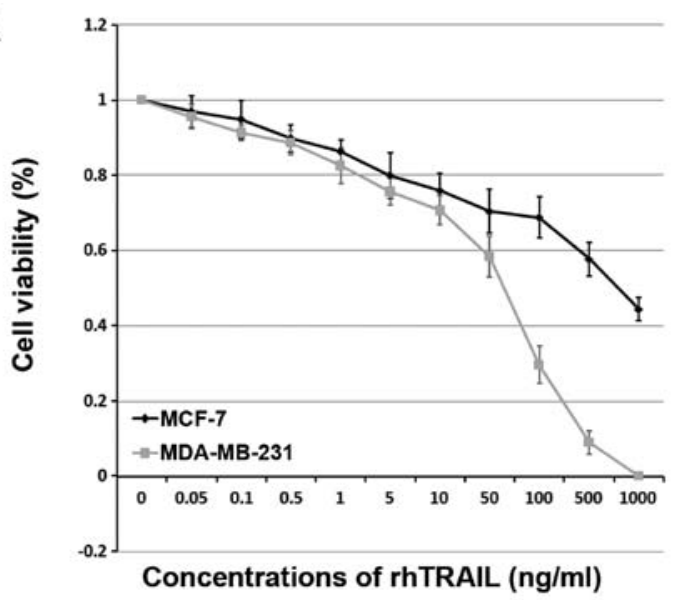

B

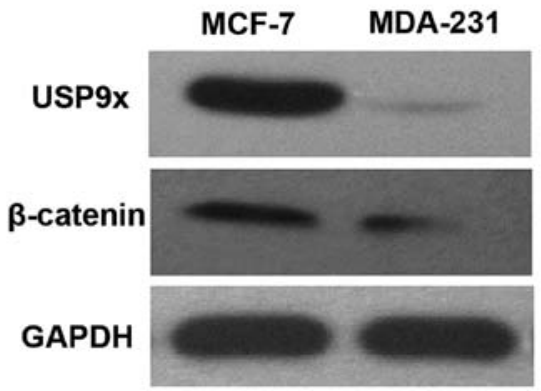

Figure 1. Expression of USP9x/ $\beta$-catenin is positively correlated with sensitivity to TRAIL. (A) The TRAIL-sensitivity of MCF-7/MDA-MB-231 cells was detected by cell cytotoxicity assay. MCF-7/MDA-MB-231 cells exposed to medium containing rhTRAIL from 0 to $1,000 \mathrm{ng} / \mathrm{ml}$ for $4 \mathrm{~h}$ were tested using the CCK- 8 assay to determine cell viability. All assays were performed in five replicates and repeated at least three times. The result is shown as a line graph (mean $\pm \mathrm{SD}$ ). The abscissa axis indicates concentrations of rhTRAIL $(\mathrm{ng} / \mathrm{ml})$, and the longitudinal axis indicates cell viability (\%); (B) The protein expression of USP9x/ $\beta$-catenin in MCF-7 and MDAMB-231 cells were detected by immunoblotting. The protein expression of USP9x/ $\beta$-catenin was normalized by GAPDH.

are 5'-tgaccacttctcatcctcacc-3' and 5'-tgggggtccacacatctc-3'; for DcR2, 5'-ggtataaagcaaaacctaaacaa-3' and 5'-catctagactacca gccttatca-3'; for OPG, 5'-gctaacctcaccttcgag-3' and 5'-tgattgga cctggttacc-3'; for USP9x, 5'-atgagaccetgctttgaacg-3' and 5'-ttcc cttctgttggatccc-3'; for $\beta$-catenin, 5'-tttgatggagttggacatgg-3' and 5'-caggacttgggaggtatcca-3'; and for GAPDH, 5'-tggaaggactcat gaccaca-3' and 5'-ttcagctcagggatgacctt-3'. Amplification products were electrophoresed on $1.8 \%$ agarose gels containing ethidium bromide for visualization under UV light. All PCR reactions were performed at least three times. PCR products were normalized to GAPDH expression.

Immunoblotting. Whole-cell extracts were prepared in RIPA lysis buffer (Biyuntian, Shanghai, China) containing protease inhibitors and were separated by 7-15\% SDS-PAGE electrophoresis under denaturing conditions, then transferred to PVDF membranes by electroblotting. The membrane was blocked with $5 \%$ skim milk in TBST buffer, incubated with the primary antibodies and reacted with HRP-conjugated secondary antibodies. The immunoreactive proteins were visualized with chemiluminescence reagent (Biyuntian). GAPDH was used to normalize protein levels. 
A

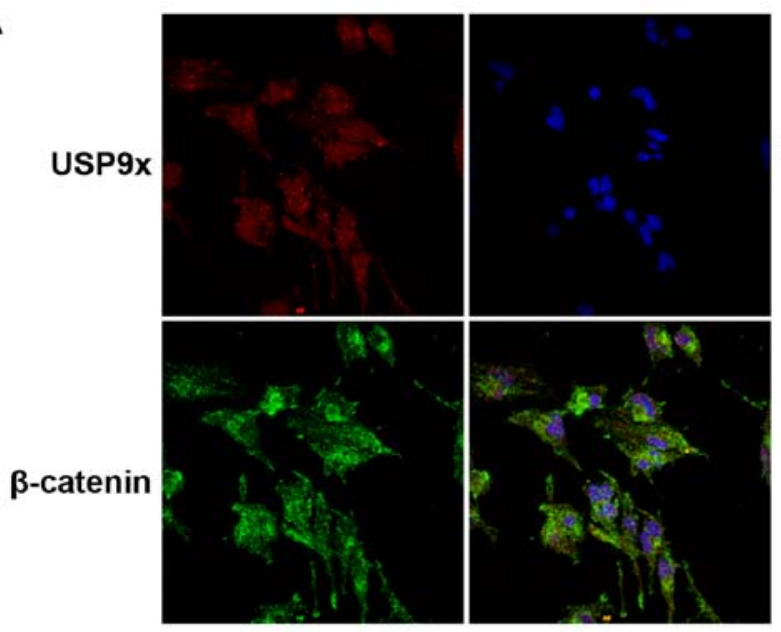

B

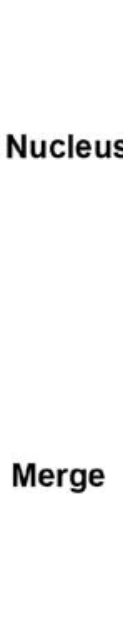

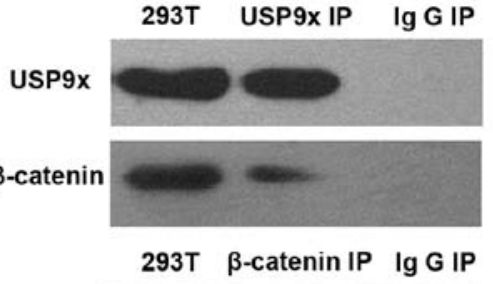

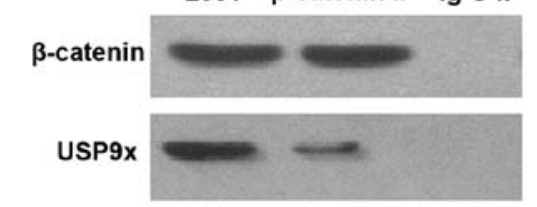

C

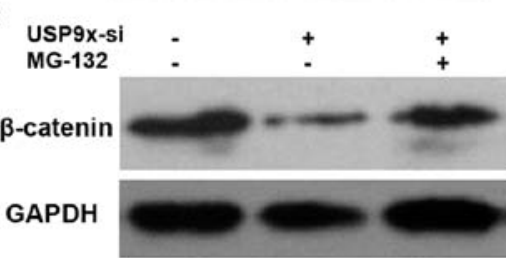

Figure 2. USP9x interacts with $\beta$-catenin and is stabilized through deubiquitination. (A) The co-localization of USP9x and $\beta$-catenin in MCF-7 cells was detected by immunofluorescence. Actual magnification of all photomicrographs is x1,000. (B) The interaction of USP9x and $\beta$-catenin was analyzed by co-immunoprecipitation in vivo. Upper panel: proteins were co-immunoprecipitated specifically with anti-USP9x antibody from HEK293T cells, and immunoblotting was adopted to detect $\beta$-catenin protein. Lower panel: proteins were co-immunoprecipitated specifically with anti- $\beta$-catenin antibody from HEK293T cells, and immunoblotting was adopted to detect USP9x protein. (C) The deubiquitinating of USP9x with $\beta$-catenin was analyzed by the inhibition of ubiquitination. MG-132 is a broad ubiquitin-proteasome inhibitor. MCF-7 cells with or without transfection of USP9x-siRNA and/or MG-132 treatment were analyzed by immunoblotting. The result of immunoblotting showed MG-132 could reverse the downregulation of $\beta$-catenin protein, which was mediated by knockdown of USP9x in MCF-7 cells.

Statistical analysis. SPSS software (version 13; IBM Corporation) was used to conduct all statistical tests. All in vitro experiments were repeated independently in triplicate. Data were pooled with mean and SE of the mean values calculated for all end points where appropriate. The two-tailed Student's t-test was used to test the significance of differences in sample means for data with normally distributed means. A p-value $<0.05$ (two-sided) was considered significant.

\section{Results}

Expression of USP9x/ $\beta$-catenin is correlated with sensitivity to TRAIL. In order to examine our hypothesis, we firstly analyzed the expression of USP9x and $\beta$-catenin protein in breast cancer MCF-7 and MDA-MB-231 cells. It has been reported that MCF-7 is resistant to TRAIL, while MDA-MB-231 is sensitive to TRAIL (17), which was also in line with our cell cytotoxicity assays to rhTRAIL (Fig. 1A). As shown, MCF-7 cells exhibited an $\mathrm{IC}_{50}$ of $>1,000 \mathrm{ng} / \mathrm{ml}$, and MDA-MB-231 cells exhibited an $\mathrm{IC}_{50}$ of $\sim 70 \mathrm{ng} / \mathrm{ml}$. To assess whether the expression of USP9x/ $\beta$-catenin is correlated with sensitivity to TRAIL in breast cancer cells, we detected the total protein expression of USP9x and $\beta$-catenin by immunoblotting (Fig. 1B). As expected, TRAIL-resistant MCF-7 cells have higher expression levels of USP9x and $\beta$-catenin than TRAIL-sensitive MDA-MB-231 cells. This result revealed that there was a positive correlation between the expression levels of USP $9 x / \beta$-catenin and sensitivity to TRAIL in breast cancer cells.

USP9x interacts and is stabilized with $\beta$-catenin through deubiquitination. USP9 $\mathrm{x}$ is a deubiquitinating enzyme. To further explore the interaction of USP9x and $\beta$-catenin, the co-localization of USP9x and $\beta$-catenin was detected by using laser confocal microscopy. As shown in Fig. 2A, both cytoplasm and cell nucleus of MCF-7 cells were positively stained for USP9 $x$ and $\beta$-catenin. The merged graph suggests that co-localization of USP9 $x$ and $\beta$-catenin existed widely in breastcancer MCF-7 cells. Then, the plasmid pCMV- $\beta$-catenin was constructed and transfected into HEK293T cells to overexpress $\beta$-catenin. To identify the interaction of USP9x and $\beta$-catenin, we analyzed proteins by co-immunoprecipitation specifically with USP9x antibody from HEK293T cells. The results of immunoblotting suggested that USP9x interacted specifically with $\beta$-catenin (Fig. 2B). Then we analyzed proteins by co-immunoprecipitation specifically with $\beta$-catenin antibody from HEK293T cells. $\beta$-catenin also interacted specifically with USP9x (Fig. 2B). In order to further confirm whether USP9x deubiquitinates $\beta$-catenin, we transfected siRNA targeting USP9x into MCF-7 cells. The result of immunoblotting showed that knockdown of USP9x declined $\beta$-catenin protein (Fig. 2C), which indicated that USP9x could stabilize $\beta$-catenin. At the same time, MG-132, is a broad ubiquitin-proteasome inhibitor elavated the total amount of $\beta$-catenin protein in MCF-7 cells with knockdown of USP9x (Fig. 2C). It indirectly confirmed that USP9 $x$ could interact and stabilize with $\beta$-catenin through deubiquitination.

DcR $1 / D c R 2 / O P G$ expression is regulated by $\beta$-catenin. It has been previously reported that expression of OPG is regulated by $\beta$-catenin/TCF in osteoblasts and colorectal cancers $(12,13)$. Here, we assessed whether $\beta$-catenin contributed to the regulation of all decoy receptor expression, including DcR1, DcR2, and OPG, in breast cancers. For this purpose, MCF-7 cells were transiently transfected with 

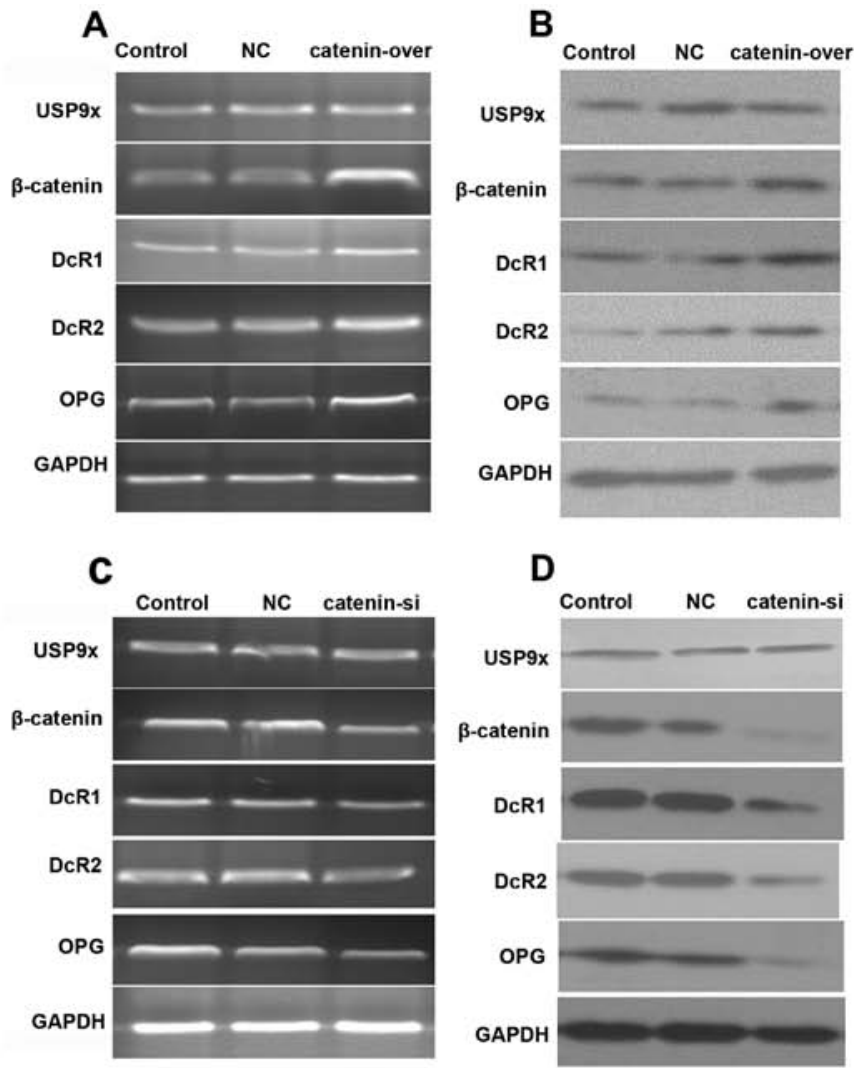

\section{E}

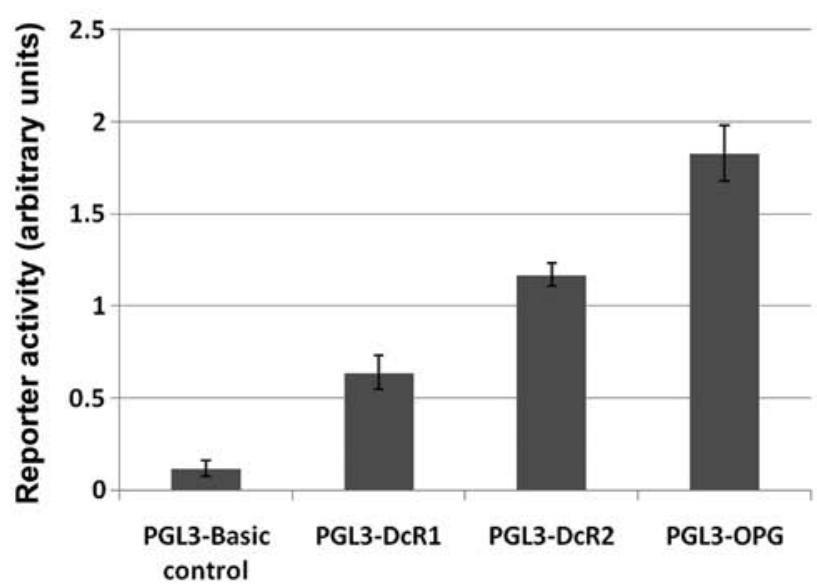

Figure 3. $\beta$-catenin regulates the expression of DcR1/DcR2/OPG. (A) The mRNA expression of USP9x, $\beta$-catenin, and decoy receptors in MCF-7 cells with or without $\beta$-catenin gene overexpression was examined by RT-PCR. (B) The protein expression of USP9x, $\beta$-catenin, and decoy receptors in MCF-7 cells with or without $\beta$-catenin gene overexpression was examined by immunoblotting. (C) The mRNA expression of USP9x, $\beta$-catenin, and decoy receptors in MCF-7 cells with or without $\beta$-catenin gene silencing was examined by RT-PCR. (D) The protein expression of USP9x, $\beta$-catenin, and decoy receptors in MCF-7 cells with or without $\beta$-catenin gene silencing was examined by immunoblotting. The mRNA/protein expression was normalized by GAPDH. (E) The transcription activity of DcR1/DcR2/OPG was detected by luciferase reporter assay. HEK293T cells were transfected with $200 \mathrm{ng}$ of a luciferase reporter gene construct for DcR1/DcR2/OPG, and expression constructs of Tcf4 and $\beta$-catenin, and $200 \mathrm{ng}$ of constitutively active $\beta$-galactosidase reporter plasmid $\mathrm{pCH} 110$, which was used to normalize luciferase activity. The result is shown as a column graph (mean $\pm \mathrm{SD})$. The longitudinal axis indicates reporter activity (arbitrary units).

overexpression plasmid pCMV- $\beta$-catenin/siRNA targeting $\beta$-catenin. It resulted in effective overexpression/silencing

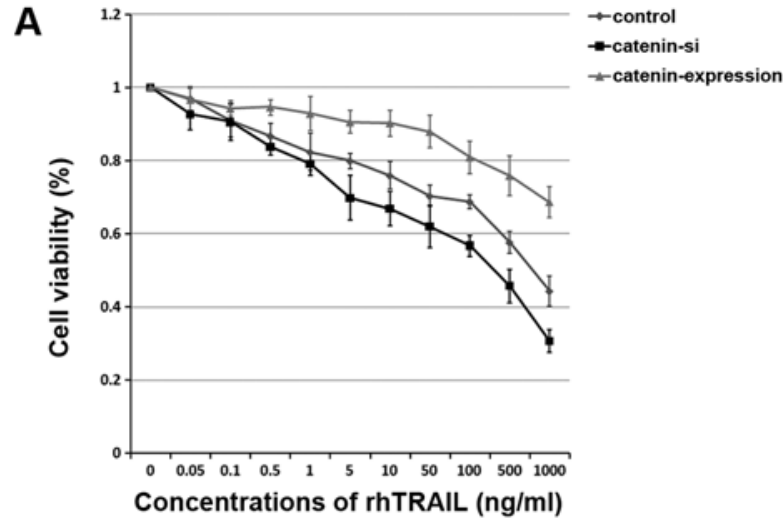

B

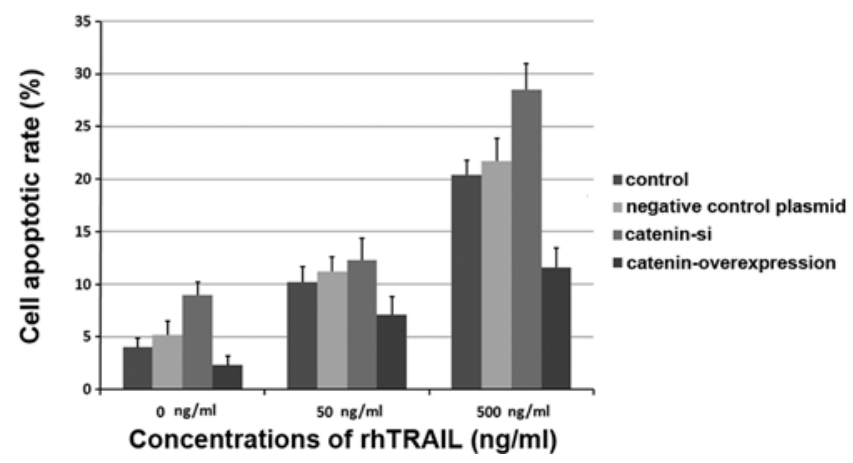

Figure 4 Expression of $\beta$-catenin is negatively correlated with sensitivity to TRAIL. (A) The viability of MCF-7 cells with or without $\beta$-catenin gene silencing/overexpression under rhTRAIL treatment at indicated concentrations for $4 \mathrm{~h}$, was tested by CCK- 8 assay. All assays were performed in five replicates and repeated at least three times. The result is shown as a line graph (mean $\pm \mathrm{SD}$ ). The abscissa axis indicates concentrations of rhTRAIL $(\mathrm{ng} / \mathrm{ml})$, and the longitudinal axis indicates cell viability (\%). (B) The apoptosis of MCF-7 cells with or without $\beta$-catenin gene silencing/overexpression and rhTRAIL treatment at indicated concentrations was analyzed by flow cytometry. Columns represent the mean data; bars, SD. The abscissa axis indicates concentrations of rhTRAIL (ng/ml), and the longitudinal axis indicates cell apoptotic rate (\%).

of $\beta$-catenin on both mRNA and protein levels (Fig. 3A-D). Increase/decrease of cellular $\beta$-catenin level coincided with a strong increase/decrease of DcR1, DcR2, and OPG mRNA expressions (Fig. 3A and C). Correspondingly, the protein expression level of DcR1/DcR2/OPG was in accordance with its respective mRNA level (Fig. 3B and D). Luciferase reporter assays showed that induction of DcR1/DcR2/OPG reporter activity was observed upon co-transfection of $\beta$-catenin and Tcf-4 (Fig. 3E). Thus, the role of $\beta$-catenin in the regulation of transcription of the human DcR1/DcR2/OPG was further confirmed.

TRAIL-induced apoptosis is regulated by expression of $\beta$-catenin. In order to investigate the effect of $\beta$-catenin in TRAIL-induced apoptosis in MCF-7 cells, we examined whether changes in expression level of $\beta$-catenin could affect MCF-7 cell sensitivity to TRAIL. After transfection with a $\beta$-catenin overexpression plasmid, the expression of $\beta$-catenin markedly increased as detected by RT-PCR (Fig. 3A) and immunoblotting analysis (Fig. 3B). As a result, the overexpression of $\beta$-catenin in MCF-7 cells led to resistance to TRAIL, which was shown by detection of viability by CCK-8 (Fig. 4A) 

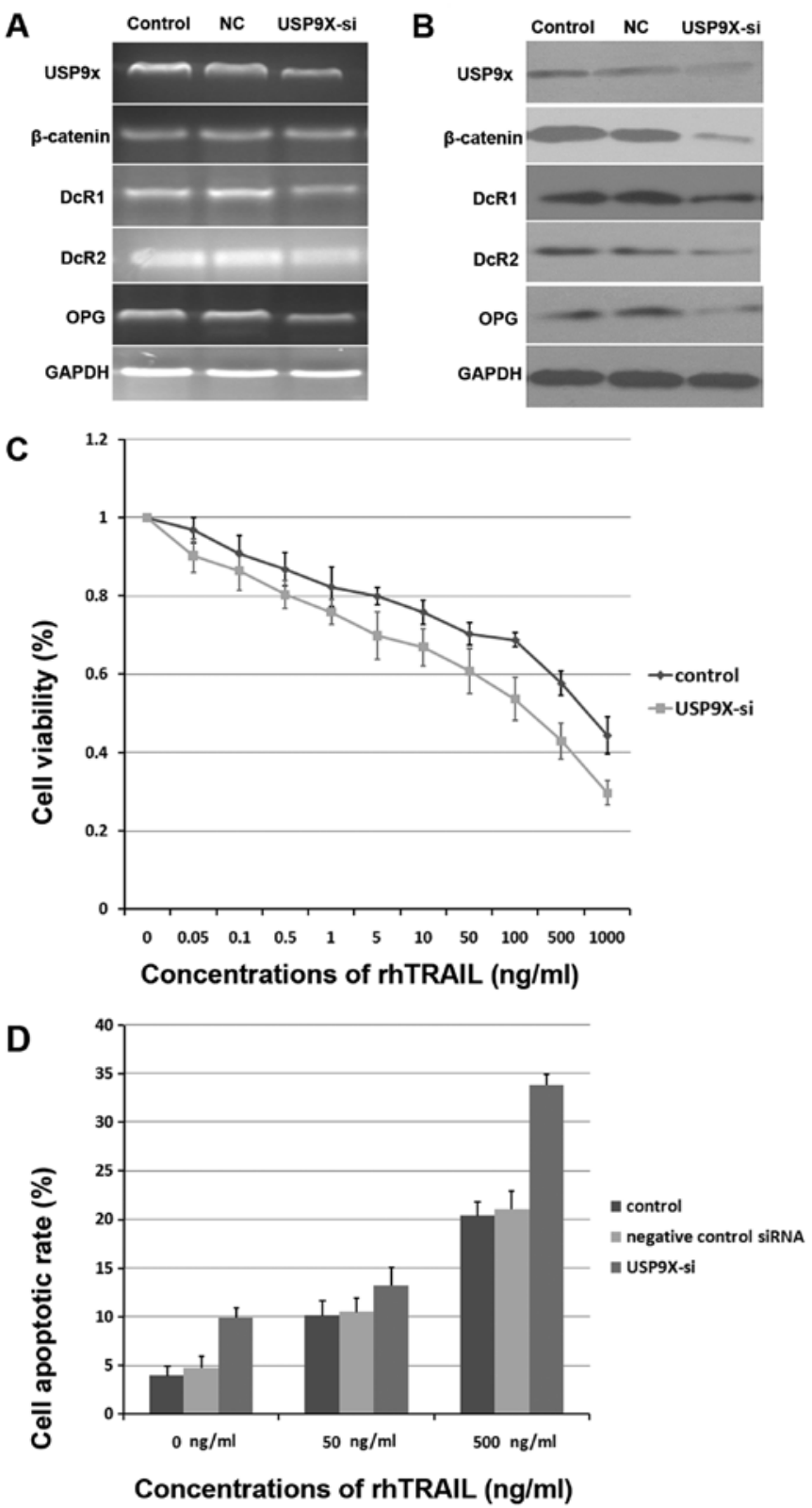

Figure 5. Silencing of USP9x increases TRAIL-sensitivity by downregulating $\beta$-catenin. (A) The mRNA expression of USP9x, $\beta$-catenin, and decoy receptors in MCF-7 cells with or without USP9x gene silencing was examined by RT-PCR. (B) The protein expression of USP9x, $\beta$-catenin and decoy receptors in MCF-7 cells with or without USP9x gene silencing was examined by immunoblotting. The mRNA/protein expression was normalized by GAPDH. (C) The viability of MCF-7 cells with or without USP9x gene silencing under rhTRAIL treatment at indicated concentrations for $4 \mathrm{~h}$, was tested by CCK-8 assay. All assays were performed in five replicates and repeated at least three times. The result is shown as a line graph (mean $\pm \mathrm{SD})$. The abscissa axis indicates concentrations of rhTRAIL (ng/ml), and the longitudinal axis indicates cell viability (\%). (D) The apoptosis of MCF-7 cells with or without USP9x gene silencing and rhTRAIL treatment at indicated concentrations was analyzed by flow cytometry. Columns represent the mean data; bars, SD. The abscissa axis indicates concentrations of rhTRAIL (ng/ml), and the longitudinal axis indicates the cell apoptotic rate (\%).

and apoptosis by flow cytometry (Fig. 4B). Similarly, after $\beta$-catenin siRNA transfection, the expression of $\beta$-catenin was significantly reduced as detected by RT-PCR (Fig. 3C) and immunoblotting analysis (Fig. 3D). On the contrary, the silencing of $\beta$-catenin in MCF-7 cells significantly elevated the sensitivity to TRAIL, which was also confirmed by detec- tion of viability by CCK-8 (Fig. 4A) and apoptosis by flow cytometry (Fig. 4B). The combined above results suggest that the expression of $\beta$-catenin plays an important role in TRAIL-induced apoptosis, which is mediated by the regulation of transcription of decoy receptors.

Silencing of USP9x reverses TRAIL-resistance by downregulating $\beta$-catenin. In order to directly evidence that USP9x affects $\beta$-catenin stability and TRAIL-induced apoptosis, we transfected siRNA targeting USP9x into MCF-7 cells. The result suggested that knockdown of USP9x significantly decreased the protein level of $\beta$-catenin, but there was almost no change in mRNA level of $\beta$-catenin (Fig. 5A and B). Furthermore, the previous results showed that MCF-7 cells were transfected with overexpression plasmid pCMV- $\beta$-catenin/siRNA targeting $\beta$-catenin, but there was no change in the mRNA or protein expression levels of USP9x (Fig. 3A-D). The above results directly indicated that USP9x was able to affect $\beta$-catenin on protein level, not on mRNA level, on the contrary, the changes in expression level of $\beta$-catenin could not affect either mRNA or protein level of USP9x. Combined with previous results, it was reconfirmed that USP9x could stabilize the protein level of $\beta$-catenin through deubiquitination. Moreover, the silencing of USP9x in MCF-7 cells significantly downregulated both mRNA and protein expression of decoy receptors, including DcR1, DcR2, and OPG (Fig. 5A and B). Ultimately, the silencing of USP9x elevated the sensitivity to TRAIL in MCF-7 cells, as confirmed by the detection of viability by CCK-8 (Fig. 5C) and apoptosis by flow cytometry (Fig. 5D). The above results evidenced our assumption that USP9x interacts and stabilizes with $\beta$-catenin through deubiquitination, resulting in overexpression of decoy receptors and TRAIL-resistance in breast cancer cells.

\section{Discussion}

Due to targeted cancer therapy having specific anticancer effects and less toxicity than conventional chemotherapy and radiotherapy, it has been an area of research focus in recent years. TRAIL is an attractive candidate that is distinguished by high selectivity for targeted cancer cells. However, analysis of a large panel of established human cancer cell lines showed that $\sim 50 \%$ of tested cancer cell lines were either partially or completely resistant to TRAIL (18). TRAIL-resistance is a major limitation for its clinical use in the treatment of human cancers. As a consequence, understanding the basis of the intrinsic resistance of cancer cells to TRAIL-mediated apoptosis is obviously important.

The cause of TRAIL-resistance is under intense scrutiny, and many possible mechanisms have been proposed. Resistance to TRAIL-induced apoptosis could be established at the membrane or intracellular level. On the level of receptor binding, the overexpression of decoy receptors, or the absence/defect of death receptors, accounts for TRAIL-resistance (19). TRAIL sensitivity may be dependent on the expression and distribution levels of TRAIL receptors (18). On the level of DISC (the death inducing signaling complex) formation, the expression of molecules that can interfere with caspase- 8 activation, such as FLIP, have been shown to play a role in TRAIL resistance (20). On the level of the intrinsic apoptosis 
pathway activation, blocking the release of apoptogenic factors can contribute to TRAIL-resistance. For example, overexpression of Bcl2 inhibited TRAIL-induced apoptosis in human lung cancer cells (21). Conversely, loss of the pro-apoptotic $\mathrm{Bcl} 2$ family member Bax rendered colon cancer cells resistant to cell death induced by TRAIL (22). In addition, it was reported that members of the apoptosis inhibitor family, cIAP, XIAP and survivin, were involved in TRAIL-resistance (23). These above mechanisms can contribute to TRAIL-resistance to varying extent in different cancer cells, and the current challenge is how to identify which of these mechanisms are responsible for the resistance in a given tumor. For TRAILinduced apoptosis, TRAIL binding to its death receptors is an upstream event during caspase activation, and the receptors are crucial in initiating apoptosis. In addition, resistance to TRAIL has been correlated with TRAIL-receptors in $~ 10 \%$ of the breast tumors examined (24). Inhibition of decoy receptor expression may make these breast tumors sensitive to TRAILinduced apoptosis and offer a new therapeutic approach for treatment of patients with breast tumors. To address the possible mechanisms of TRAIL-resistance in breast cancer cells, we chose TRAIL-resistant breast cancer MCF-7 cells to explore.

The gene promoter upstream regions of decoy receptors, such as DcR1, DcR2, and OPG, are enriched in binding sites for TCF/LEF transcription factor. Here, we assessed whether $\beta$-catenin contributed to the regulation of decoy receptor transcription. Luciferase reporter assays showed induction of DcR 1/ DcR2/OPG reporter activity observed upon co-transfection of $\beta$-catenin and Tcf-4 (Fig. 3E). Overexpression of $\beta$-catenin in MCF-7 cells, subsequently resulted in a strong increase of DcR1, DcR2, and OPG mRNA expressions (Fig. 3A), then led to resistance to TRAIL (Fig. 4). The important role of $\beta$-catenin in sensitivity to TRAIL was confirmed in breast cancer cells, and was mediated by regulating transcription of the human DcR1/DcR2/OPG.

However, cytoplasmic $\beta$-catenin is maintained at low levels in mature normal cells most of the time (14). In the absence of Wnt/ $\beta$-catenin signaling pathway, free $\beta$-catenin is sequestered in a complex that consists of the adenomatous polyposis coli (APC) tumor suppressor, Axin, glycogen synthase kinase- $3 \beta$ (GSK3 $\beta$ ), and casein kinase 1 (CK1). This complex formation induces the phosphorylation of $\beta$-catenin by CK1 and GSK3 $\beta$, which results in the ubiquitination and the subsequent degradation of $\beta$-catenin by the $26 \mathrm{~S}$ proteasome (25). The mechanisms of $\beta$-catenin overexpression remained elusive. Our results showed that TRAIL-resistant MCF-7 cells have higher expression levels of $\beta$-catenin than TRAIL-sensitive MDA-MB-231 cells, as well as the deubiquitinating enzyme USP9x (Fig. 1). It also was reported that Fam, which is the mouse homologous protein of USP9x, interacted with $\beta$-catenin, and the Fam-binding site of $\beta$-catenin mapped to the region close to the APC or Axin-binding site of $\beta$-catenin (16). This result may explain the positive correlation between the expression levels of USP $9 x / \beta$-catenin and sensitivity to TRAIL in breast cancer cells. Subsequently, our studies confirmed it in human breast cancer cells showing that USP9x was co-localizated with $\beta$-catenin (Fig. 2A), and USP9x interacted with $\beta$-catenin (Fig. $2 \mathrm{~B}$ ) and inhibited the degradation of $\beta$-catenin (Fig. 5A and B) through the deubiquitination of $\beta$-catenin (Fig. 2C).
Taken together, our studies indicate that USP9x interacts and stabilizes with $\beta$-catenin by deubiquitination, which results in the accumulation of cytoplasmic $\beta$-catenin, then $\beta$-catenin translocates into the nucleus where it interacts with $\mathrm{TCF} / \mathrm{LEF}$ to induce the expression of decoy receptors, finally leading to resistance to TRAIL in breast cancer cells. Our results offer new insights into the mechanisms of resistance to TRAIL in breast cancer cells. The regulation of USP9x could potentially be a plausible method for improvement of TRAIL-sensitivity, so USP9x could also potentially be a therapeutic target for TRAIL-resistant breast cancers.

\section{Acknowledgements}

This study was supported by grants from the National Natural Science Foundation of China (no. 81372498) and the Youth Science Foundation of Zhongnan Hospital, Wuhan University (no. 2014A07).

\section{References}

1. Wiley SR, Schooley K, Smolak PJ, Din WS, Huang CP, Nicholl JK, Sutherland GR, Smith TD, Rauch C, Smith CA, et al: Identification and characterization of a new member of the TNF family that induces apoptosis. Immunity 3: 673-682, 1995.

2. Amm HM, Oliver PG, Lee CH, Li Y and Buchsbaum DJ: Combined modality therapy with TRAIL or agonistic death receptor antibodies. Cancer Biol Ther 11: 431-449, 2011.

3. Wakelee HA, Patnaik A, Sikic BI, Mita M, Fox NL, Miceli R, Ullrich SJ, Fisher GA and Tolcher AW: Phase I and pharmacokinetic study of lexatumumab (HGS-ETR2) given every 2 weeks in patients with advanced solid tumors. Ann Oncol 21: 376-381, 2010.

4. Herbst RS, Eckhardt SG, Kurzrock R, Ebbinghaus S, O'Dwyer PJ, Gordon MS, Novotny W, Goldwasser MA, Tohnya TM, Lum BL, et al: Phase I dose-escalation study of recombinant human Apo2L/TRAIL, a dual proapoptotic receptor agonist, in patients with advanced cancer. J Clin Oncol 28: 2839-2846, 2010.

5. Pan G, O'Rourke K, Chinnaiyan AM, Gentz R, Ebner R, Ni J and Dixit VM: The receptor for the cytotoxic ligand TRAIL. Science 276: 111-113, 1997.

6. Mérino D, Lalaoui N, Morizot A, Schneider P, Solary E and Micheau O: Differential inhibition of TRAIL-mediated DR5-DISC formation by decoy receptors 1 and 2. Mol Cell Biol 26: 7046-7055, 2006.

7. Wu F, Hu Y, Long J, Zhou YJ, Zhong YH, Liao ZK, Liu SQ, Zhou FX, Zhou YF and Xie CH: Cytotoxicity and radiosensitization effect of TRA-8 on radioresistant human larynx squamous carcinoma cells. Oncol Rep 21: 461-465, 2009.

8. Johnston JB, Kabore AF, Strutinsky J, Hu X, Paul JT, Kropp DM, Kuschak B, Begleiter A and Gibson SB: Role of the TRAIL/APO2-L death receptors in chlorambucil- and fludarabine-induced apoptosis in chronic lymphocytic leukemia. Oncogene 22: 8356-8369, 2003.

9. MacDonald BT, Tamai K and He X: Wnt/beta-catenin signaling: Components, mechanisms, and diseases. Dev Cell 17: 9-26, 2009.

10. Zhang S, Li Y, Wu Y, Shi K, Bing L and Hao J: Wnt/ $\beta$-catenin signaling pathway upregulates c-Myc expression to promote cell proliferation of P19 teratocarcinoma cells. Anat Rec (Hoboken) 295: 2104-2113, 2012.

11. Tanaka SS, Kojima Y, Yamaguchi YL, Nishinakamura R and Tam PP: Impact of WNT signaling on tissue lineage differentiation in the early mouse embryo. Dev Growth Differ 53: 843-856, 2011.

12. Glass DA II, Bialek P, Ahn JD, Starbuck M, Patel MS, Clevers H, Taketo MM, Long F, McMahon AP, Lang RA, et al: Canonical Wnt signaling in differentiated osteoblasts controls osteoclast differentiation. Dev Cell 8: 751-764, 2005.

13. De Toni EN, Thieme SE, Herbst A, Behrens A, Stieber P, Jung A, Blum H, Göke B and Kolligs FT: OPG is regulated by beta-catenin and mediates resistance to TRAIL-induced apoptosis in colon cancer. Clin Cancer Res 14: 4713-4718, 2008. 
14. Orford K, Crockett C, Jensen JP, Weissman AM and Byers SW: Serine phosphorylation-regulated ubiquitination and degradation of beta-catenin. J Biol Chem 272: 24735-24738, 1997.

15. Rott R, Szargel R, Haskin J, Bandopadhyay R, Lees AJ, Shani $\mathrm{V}$ and Engelender $\mathrm{S}$ : $\alpha$-Synuclein fate is determined by USP9X-regulated monoubiquitination. Proc Natl Acad Sci USA 108: 18666-18671, 2011.

16. Taya S, Yamamoto T, Kanai-Azuma M, Wood SA and Kaibuchi K: The deubiquitinating enzyme Fam interacts with and stabilizes beta-catenin. Genes Cells 4: 757-767, 1999.

17. Park S, Shim SM, Nam SH, Andera L, Suh N and Kim I: CGP74514A enhances TRAIL-induced apoptosis in breast cancer cells by reducing X-linked inhibitor of apoptosis protein. Anticancer Res 34: 3557-3562, 2014

18. Ouyang W, Yang C, Liu Y, Xiong J, Zhang J, Zhong Y, Zhang G, Zhou F, Zhou Y and Xie C: Redistribution of DR4 and DR5 in lipid rafts accounts for the sensitivity to TRAIL in NSCLC cells. Int J Oncol 39: 1577-1586, 2011

19. Wu Q, Zheng Y, Chen D, Li X, Lu C and Zhang Z: Aberrant expression of decoy receptor 3 in human breast cancer: Relevance to lymphangiogenesis. J Surg Res 188: 459-465, 2014.

20. Wang W, Zhou J, Shi J, Zhang Y, Liu S, Liu Y, Zheng D and Ross SR: Human T-cell leukemia virus type 1 Tax-deregulated autophagy pathway and c-FLIP expression contribute to resistance against death receptor-mediated apoptosis. J Virol 88 2786-2798, 2014.
21. Sun SY, Yue P, Zhou JY, Wang Y, Choi Kim HR, Lotan R and Wu GS: Overexpression of BCL2 blocks TNF-related apoptosis-inducing ligand (TRAIL)-induced apoptosis in human lung cancer cells. Biochem Biophys Res Commun 280: 788-797, 2001.

22. Deng Y, Lin Y and Wu X: TRAIL-induced apoptosis requires Bax-dependent mitochondrial release of Smac/DIABLO. Genes Dev 16: 33-45, 2002

23. Cummins JM, Kohli M, Rago C, Kinzler KW, Vogelstein B and Bunz F: X-linked inhibitor of apoptosis protein (XIAP) is a nonredundant modulator of tumor necrosis factor-related apoptosis-inducing ligand (TRAIL)-mediated apoptosis in human cancer cells. Cancer Res 64: 3006-3008, 2004

24. Shin MS, Kim HS, Lee SH, Park WS, Kim SY, Park JY, Lee JH, Lee SK, Lee SN, Jung SS, et al: Mutations of tumor necrosis factor-related apoptosis-inducing ligand receptor 1 (TRAIL-R1) and receptor 2 (TRAIL-R2) genes in metastatic breast cancers. Cancer Res 61: 4942-4946, 2001.

25. King TD, Suto MJ and Li Y: The Wnt/ $\beta$-catenin signaling pathway: A potential therapeutic target in the treatment of triple negative breast cancer. J Cell Biochem 113: 13-18, 2012. 